\title{
Dialysis dose in end-stage renal disease patients undergoing regular hemodialysis at Ibn-Sena and Al- Nao hospitals in Sudan
}

\author{
Essamaddin A Ibrahim* ${ }^{*}$ \\ Department of Internal Medicine, University of Science and Technology
}

\section{A R T I C L E I N F O}

Article Type:

Brief Report

Article History:

Received: 14 September 2018

Accepted: 8 January 2019

Published online: 7 June 2019

\section{Keywords:}

End stage renal disease,

Hemodialysis, Chronic kidney

disease, Glomerular filtration

rate, Vascular access, Renal

replacement therapy

\begin{abstract}
A B S T R A C T
Introduction: Ne Hemodialysis (HD) plays a fundamental role in the treatment of end-stage renal disease (ESRD) patients. A minimal duration between 9 to 12 hours per week is required for maintaining adequate HD to restore excretory function of the kidney.

Objectives: To measure the average duration of HD among Sudanese patients, and compare with the universal recommended duration.

Patients and Methods: A cross-sectional, descriptive study at IBN-SENA hospital and ALNAO teaching hospital was conducted. All patients were subjected to full medical history and examination to identify their age, gender, original kidney disease and duration and route of HD. Results: A total of 121 ESRD patients under regular HD participated in this study. The majority [78 (64.5\%)] had an average duration of $\mathrm{HD}$ of $8 \mathrm{~h} / \mathrm{wk}$.

Conclusion: A large proportion of our patients on $\mathrm{HD}$ are below target dialysis dose; mainly due to reducing in the number of sessions per week and average duration per each session.
\end{abstract}

Implication for health policy/practice/research/medical education:

In a cross-sectional, descriptive study on 121 hemodialysis patients in Sudan, we found a large proportion of our patients are below target dialysis dose; mainly due to reducing in the number of sessions per week and average duration per each session. Please cite this paper as: Ibrahim EA. Dialysis dose in end-stage renal disease patients undergoing regular hemodialysis at IbnSena and Al-Nao hospitals in Sudan. J Nephropharmacol. 2020;9(1):e10. DOI: 10.15171/npj.2020.10.

\section{Introduction}

Chronic kidney disease $(\mathrm{CKD})$ is an irreversible deterioration in renal function over months to years. CKD is classified based on estimated glomerular filtration rate (eGFR) into five stages. End-stage renal disease (ESRD) is the CKD of stage 5 at which the patient starts renal replacement therapy (RRT) which includes hemodialysis (HD), peritoneal dialysis or kidney transplantation $(1,2)$. CKD affects $5 \%-10 \%$ of the world population $(3,4)$, led to 956000 deaths globally in 2013 up from 409000 deaths in 1990 (5). Both diabetes and hypertension are important causes of ESRD, since screening of at-risk patients is important to delay the progression of CKD (6). HD is the most commonly used in ESRD patients. Vascular access for HD must be established by a fistula, graft or catheter through which blood obtained for $\operatorname{HD}(7,8)$. The purpose of $\mathrm{HD}$ is to restore excretory function of the kidney and to sustain normal electrolyte concentration and fluid balance (9). For the majority of the patients is a minimal duration between 9 to 12 hours per week to maintain adequate HD (10).

\section{Objectives}

The aim of this study is to measure the average duration of HD among Sudanese patients to compare with the universal recommendation.

Patients and Methods

Study setting

This is a cross-sectional, descriptive study undertaken conducted between July to December 2017 at Ibn-Sena hospital center of renal disease and transplantation and AL-Nao teaching hospital center of renal dialysis.

The study population was all ESRD patients on regular 
HD for at least 12 weeks. Patients under the age of 18 years were excluded.

All patients subjected to full medical history and examination to identify their age, gender, original kidney disease, duration and route of HD, number of sessions per week and average duration of each session.

\section{Ethical approval}

The protocol of the study was approved by ethical committee of the Sudan medical specialization board. The current study was performed according to the Institutional Committee for the Protection of human subjects, which was adopted by the $18^{\text {th }}$ World Medical Assembly, Helsinki, Finland and its later amendments. Written informed consents were obtained from the parents of both patients and controls.

\section{Statistical analysis}

Data was collected through a structured questionnaire that contained open and close-ended questions. The data was analyzed by IBM SPSS statistics 23 . $P$ value $<0.05$ were taken as significant.

\section{Results}

One hundred and twenty-one of ESRD patients under regular HD participated in this study. There were 80 (66.1\%) males and 41(33.9\%) females. The majority [34(28.1\%)] had an age group between 50-59 years (Table 1).

Regarding duration of HD, most of patients [63(52.1\%)] had dialysis duration between one to five years. Vascular access for dialysis was achieved by arteriovenous fistula in $94(77 \%)$ patients and by semi-permanent catheter in $23(19 \%)$ patients since only $4(3.3 \%)$ patients had temporary catheter (Table 2). In the comorbidity, hypertension was found in $43(35.5 \%)$ patients followed by diabetes in $19(15.7 \%)$ patients (Table 3$)$. The majority of patients [78(64.5\%)] had average duration of HD of $8 \mathrm{~h} /$ wk, whereas $33(27.3 \%)$ patients had average duration of $\mathrm{HD}$ around $6 \mathrm{~h} / \mathrm{wk}$ and only $9(7.4 \%)$ patients had average duration of $12 \mathrm{~h} / \mathrm{wk}$ (Table 4$)$.

Table 1. Age and gender distribution in the study population

\begin{tabular}{lll}
\hline & No. & Percent \\
\hline Age $(\mathrm{y})$ & & \\
18 years to 29 years & 14 & 11.6 \\
30 years to 39 years & 21 & 17.4 \\
40 years to 49 years & 24 & 19.8 \\
50 years to 59 years & 34 & 28.1 \\
60 years or more & 28 & 23.1 \\
Gender & & \\
Male & 80 & 66.1 \\
Female & 41 & 33.9 \\
\hline
\end{tabular}

Table 2. Duration of hemodialysis and vascular access in the study population

\begin{tabular}{lcc}
\hline & Number & Percent \\
\hline Duration of hemodialysis & 13 & 10.7 \\
3 months to 11 months & 63 & 52.1 \\
1 year to 5 years & 45 & 37.2 \\
More than 5 years & & \\
Vascular access & 94 & 77.7 \\
Arteriovenous fistula & 23 & 19 \\
Semi-permanent catheter & 4 & 3.3 \\
Temporary catheter & & \\
\hline
\end{tabular}

Table 3. The cause of ESRD in study population

\begin{tabular}{lcc}
\hline & Number & Percent \\
\hline Renal failure cause & & \\
Diabetes & 19 & 15.7 \\
Hypertension & 43 & 35.5 \\
Obstructive uropathy & 13 & 10.7 \\
PCKD & 3 & 2.5 \\
Others & 43 & 35.5 \\
\hline
\end{tabular}

Table 4. Dialysis characteristics (rate, average duration of each session and hemodialysis dose per week)

\begin{tabular}{lll}
\hline & Number & Percent \\
\hline Rate of HD session per week & 1 & \\
One session & 108 & 89.8 \\
Two sessions & 12 & 9.9 \\
Three sessions & & \\
Average duration of each session & 37 & 30.6 \\
Two to three hours & 84 & 69.4 \\
Three to four hours & & \\
Hemodialysis dose per week & 1 & 0.8 \\
Three hours per week & 33 & 27.3 \\
Six hours per week & 78 & 64.5 \\
Nine hours per week & 9 & 7.4 \\
Twelve hours per week & & \\
\hline
\end{tabular}

\section{Discussion}

The majority of ESRD patients undergoing regular $\mathrm{HD}$ are males with a risk factor for developing CKD and ESRD in our country.

Hypertension plays a major role as the most common cause of ESRD in the study population. Data in this study was concordant with the study by Banaga et al (11).

Only 9 patients (7.4\%) had adequate HD dose and the remaining (92\%) had dialysis dose less than the universal recommendation. Several factors lead to this gap. Firstly; 180 patients $(89.3 \%)$ undergo two sessions rather than three per week due to restricted resources and lack of sufficient number of HD machines to cover ESRD patients. Additionally, more than $30 \%$ of the patients have only average 2-3 hours per each session. This could 
be due to improper HD which affects perpetuation of the sessions. In addition, traffic and transportation inefficiency is responsible for patient's arrival behind the schedule, since some patients had a tendency to terminate the dialysis session before reaching the target hours due to individualized reasons.

\section{Conflicts of interest}

The author declares that he has no conflicts interest.

\section{Ethical considerations}

Ethical issues (including plagiarism, data fabrication, double publication) have been completely observed by the author.

\section{Author's contribution}

EAI is the single author of the manuscript.

\section{Funding/Support}

None.

\section{References}

1. National Kidney Foundation. K/DOQI clinical practice guidelines for chronic kidney disease. Am J Kidney Dis. 2002;39(2 Suppl 1):S1-266.

2. KDIGO: Kidney Disease Improving Global Outcomes. KDIGO Clinical Practice Guideline for the Diagnosis, Evaluation, Prevention, and Treatment of Chronic Kidney Disease-Mineral and Bone Disorder (CKD-MBD). Kidney Int Suppl. 2009 Aug;(113):S1-130. doi: 10.1038/ki.2009.188.

3. Eknoyan G, Lameire N, Barsoum R, Eckardt KU, Levin A,
Levin N, et al. The burden of kidney disease: improving global outcomes. Kidney Int. 2004;66:1310-14

4. Martínez-Castelao A, Górriz JL, Segura-de la Morena J, Cebollada J, Escalada J, Esmatjes E, Fácila L, et al. Consensus document for the detection and management of chronic kidney disease. Nefrologia. 2014;34:243-62. doi: 10.3265/Nefrologia.pre2014.Feb.12455.

5. GBD 2013 Mortality and Causes of Death Collaborators. Global, regional, and national age-sex specific all-cause and cause-specific mortality for 240 causes of death, 1990-2013: a systematic analysis for the Global Burden of Disease Study 2013. Lancet. 2014;385:117-71

6. Plantinga LC, Tuot DS, Powe NR. Awareness of chronic kidney disease among patients and providers". Adv Chronic Kidney Dis. 2010;17:225-36

7. Pendse S, Singh A, Zawada E. Initiation of Dialysis. In: Handbook of Dialysis. 4th ed. New York, NY; Lippin-cott Williams \& Wilkins: 2008:14-21

8. Bennett WM, Henrich WL, Schrier RW. Atlas of Diseases of the Kidney. Volume 5. Wiley-Blackwell; 1999.

9. National Kidney and Urologic Diseases Information Clearinghouse guidance. Kidney Failure: Choosing a Treatment That's Right for You. National Institute of Diabetes and Digestive and Kidney Diseases;2007.

10. J. Larry Jameson JL, Loscalzo J. Harrison's Nephrology and Acid-Base Disorders. 3rd ed. McGraw-Hill Education; 2016.

11. Banaga AS, Mohammed EB, Siddig RM, Salama DE, Elbashir SB2, Khojali MO. Causes of end stage renal failure among haemodialysis patients in Khartoum State/Sudan. BMC Res Notes. 2015;8:502. doi: 10.1186/s13104-0151509-x.

Copyright $\odot 2020$ The Author(s); Published by Published by Society of Diabetic Nephropathy Prevention. This is an open-access article distributed under the terms of the Creative Commons Attribution License (http://creativecommons.org/licenses/by/4.0), which permits unrestricted use, distribution, and reproduction in any medium, provided the original work is properly cited. 